\title{
Free hand proprioception is well calibrated to verbal estimates of slanted surfaces
}

\author{
Dennis M. Shaffer ${ }^{1}$ - Ally Taylor ${ }^{1}$
}

Published online: 8 December 2016

(C) The Psychonomic Society, Inc. 2016

\begin{abstract}
We investigate the relationship between verbal and hand proprioception of slant. In Experiment 1 we demonstrate that verbally estimating free hand orientation produces overestimates by a factor of 1.67 . These values are similar to those seen for verbal overestimates of slanted surfaces. In Experiment 2, participants positioned their hand to a ramp at 1 of 4 different orientations, and then verbally estimated the orientation of either their hand or the ramp. We show that verbal estimates of the ramp are a product of the orientation of their hand and the perception of the orientation of their hand. We discuss how this work is consistent with the proprioception calibration hypothesis that proposes that similar biases exist in both verbal estimates of visually perceived slant and proprioceptively perceived hand orientation and how this may explain free hand estimates to outdoor hills that are greater than actual hill orientation by a factor of $\sim 2$, but are still less than verbal (over)estimates.
\end{abstract}

Keywords Slant perception $\cdot$ Spatial orientation $\cdot$ Pitch

An abundance of work over the last 2 decades has shown that people verbally overestimate the slant of visually perceived geographical, virtual, and man-made hills by between $5^{\circ}$ and $25^{\circ}$ (Durgin \& Li, 2011; Hajnal, Abdul-Malak, \& Durgin, 2011; Li \& Durgin, 2010; Proffitt, Bhalla, Gossweiler, \& Midgett, 1995; Shaffer \& Flint, 2011). In much of this work, verbal estimates of slant are accompanied by another method for measuring perceived slant, called haptic matching. Haptic

Dennis M. Shaffer

shaffer.247@osu.edu

1 Department of Psychology, The Ohio State University-Mansfield, 1760 University Drive, Mansfield, OH 44906, USA matching estimates are sometimes made using a palm board -where people rest their hand on and rotate the palm board up from horizontal to match the palm board to their visual perception of the slope of the hill (e.g., Bhalla \& Proffitt, 1999; Durgin, Hajnal, Li, Tonge, \& Stigliani, 2010; Proffitt et al., 1995; Taylor-Covill \& Eves, 2013).

Historically, palm board estimates have been closer to the true inclinations of hills (especially for hills at or above $\sim 10^{\circ}$ ). This work has documented that the different measures of palm boards and verbal estimation give different values for slanted surfaces, leading to the conclusion that there are two representations of slant - one for action and a separate representation for perception (Bhalla \& Proffitt, 1999; Creem \& Proffitt, 1998; Proffitt et al., 1995). However, our recent work has demonstrated a fundamental issue that explains why two different values typically given for palm board and verbal/visual matching estimates express a similar perceptual representation of slanted surfaces (Shaffer, Taylor, McManama, et al., 2016). We articulated that the fundamental problem in studies measuring palm board and verbal estimates is that the measure (either reproducing a verbally given angle or the orientation of a slanted surface with an unseen hand or verbally or visually estimating a visually perceived surface) has always been confounded with the surface (either using a palm board or a hill/ramp). Although reproduction had been exclusively used with palm boards in these studies, verbal estimation or visual matching had been exclusively used with hills/ramps. In our work, we showed that verbally estimating palm board orientation produces overestimates equivalent to verbal overestimates of similarly slanted surfaces, while reproducing the orientation of a surface of a ramp to verbally given angles produces estimates equivalent to palm board estimates of those same slanted surfaces. Perhaps a simpler way to think about this is if slant in general is overestimated, and you show observers a slanted surface and have them match their hand to it, 
you would expect them to stop short of the perceived angle, as the hand that you are matching with feels and looks steeper than it actually is. Conversely, if people verbally give a specific value of the orientation of a hill (or their own hand), one would expect overestimates as the hill or hand looks or feels steeper than it truly is. The findings point to the fact that whether using a reproduction measure (i.e., the palm board) or a verbal estimation measure (i.e., a verbal estimate of a viewed slanted surface), the perceptual representation of space is the same. Thus, we found that a palm board estimate, or the perceptual representation of a slanted surface as measured via the palm board, is well calibrated to the perceptual representation of the same slanted surface as measured via a verbal estimate (Shaffer, Taylor, Thomas, et al., 2016).

This work joins a growing body of work that is consistent with the proprioception calibration hypothesis, which proposes a similar calibration between verbal estimates of visually perceived slant and proprioceptively perceived orientation (Li \& Durgin, 2012). For instance, palm board estimates can be biased because of wrist flexion and anchoring issues (e.g., Durgin, Hajnal, et al., 2010; Durgin, Li, et al., 2010; Shaffer, McManama, Swank, Williams, \& Durgin, 2014), which we know they are, and our perception of the orientation of our hand and a ramp/hill can be gross overestimations of the true inclination of the ramp/hill. However, as long as we overestimate our hand and the ramp/hill in the same way (by the same magnitude), our actions will be aligned the way they need to be in order to appropriately interact with surfaces. More recent work is also consistent with calibrating proprioceptive and visual perception showing that nonverbal methods match verbal estimates regarding slant. For instance, measures of matching extent and remote haptic perception have been shown to be supportive of a single underlying representation that is exaggerated to the same extent as the results are for verbal and visual matching estimates (Li \& Durgin, 2010; Shaffer \& McManama, 2015). Li and Durgin (2010) had one group of people compare the relative length of a frontal extent to that of an extent that was placed up a virtual hill (tilted back in depth). The other group gave verbal estimates of the same hill. The implicit slant measure gave the same results of the verbal estimates - both showed the same exaggeration of all slants. Shaffer and McManama (2015) used a remote haptic device in which the participant holds one end of a wooden dowel while exploring an $\sim 1$-m inclined surface. The remote haptic task allows exploration of the surface itself using proprioceptive feedback and is also an action-based measure very good at affording appropriate action upon the same slanted surface on which it is used (Fitzpatrick, Carello, Schmidt, \& Corey, 1994; Malek \& Wagman, 2008). The slope of the gain of estimates across six inclinations using the remote haptic task was exactly the same slope (ratio of estimate: actual inclination) that has been shown for verbal estimates of virtual and geographical hills, nonverbal matching estimates, haptic estimates using a finger to explore slanted surfaces, and estimates given when pitching people backward with an inversion table (Durgin \& Li, 2012; Hajnal et al., 2011; Shaffer, Taylor, Thomas, et al., 2016). This work lends support for the idea that our visual system is scaled in the same way as our haptic system, resulting in a common underlying perceptual experience.

Haptic matching estimates are also sometimes made using a free hand measure (Bridgeman \& Hoover, 2008; Durgin, Hajnal, et al., 2010; Durgin, Li, et al., 2010; Li \& Durgin, 2011; Shaffer et al., 2014). This consists of holding one's unseen hand and/or forearm parallel to the slope. Although free hand estimates of slant within reach have been shown to be fairly accurate (e.g., Durgin, Hajnal, et al., 2010, Experiments 1 \& 3; Durgin, Li, \& Hajnal, 2010, Experiment 2; Li \& Durgin, 2011; Li \& Durgin, 2012, Experiments 1 \& 2), free hand estimates of visually perceived surfaces that are out of reach including outdoor hills suggest that hills appear much steeper than their physical inclination, with free hand estimates overestimating slant of out of reach surfaces, but not as much as verbal estimates do (Bridgeman \& Hoover, 2008; Hecht, Shaffer, Keshvarz, \& Flint, 2014; Shaffer et al., 2014; Shaffer, McManama, \& Durgin, 2015).

In the current work, we sought to attempt to model the relationship of free hand estimates of slanted surfaces out of reach to verbal estimates of perceived slant, similar to how Shaffer, Taylor, McManama, et al. (2016) mapped out the relationship between palm board and verbal estimates. $\mathrm{Li}$ and Durgin (2011) have proposed that free hand estimates provide a bridge to estimates of the orientation of outdoor hills by providing what they call a "near surface equivalent"-the near surface orientation that would be judged to be equivalent in slant. They found that the gain of the best-fit line relating free hand orientation to verbal estimates for outdoor hills was almost identical to that relating orientations of near surfaces to verbal estimates of those same orientations. We sought to extend this work by mapping out the relationship between actual free hand orientation and perceived free hand orientation to verbal estimates of perceived slant of a surface that was out of reach ( $\sim 1 \mathrm{~m}$ away). Because free hand estimates are well calibrated for near surfaces and are reliable across a wide range of angles (Durgin, Hajnal, et al., 2010; Durgin, Li, et al., 2010; Shaffer et al., 2015), we expect that verbal estimates of surface orientation that is out of reach should be a product of actual orientation of the hand held freely in the air and perceived orientation of the hand while it is held freely in the air, consistent with the proprioception calibration hypothesis.

We first tested whether, when asked to verbally estimate their hand while freely moved in the air, at what orientation participants would estimate it to be positioned. We then tested whether verbal estimates of the orientation of a ramp positioned out of reach of participants ( $1 \mathrm{~m}$ away) were a product of, or could be predicted from, free hand orientation and 
verbal estimates of free hand orientation by having participants orient their free hand to different surface orientations of a ramp, and then verbally estimate either the orientation of their hand or a ramp.

\section{Experiment 1}

Experiment 1 was performed to investigate whether free hand proprioception is overestimated, by what factor it is overestimated, and whether this overestimation is similar to verbal overestimates of slope by directly asking people to estimate at what angle their hand is oriented when placed at various angles.

\section{Method}

\section{Participants}

Twenty undergraduates from The Ohio State University at Mansfield (12 male) participated in fulfillment of an Introductory Psychology requirement.

\section{Materials}

A flat piece of wood measuring $15.24 \mathrm{~cm} \times 8.89 \mathrm{~cm}$ and weighing $0.18 \mathrm{~kg}$ was placed on the palm side of the participants' self-rated dominant hand. This was attached securely to the hand of the observer by elastic straps. Measurements of forearm orientation were taken using an Accuremote ${ }^{\mathrm{TM}}$ precision angle measurement gauge that was placed on the piece of wood attached to their hand. Participants could not see their hand when making their settings. In the eyes-open task, a vertical screen blocking the participants' view of their hand was adjusted to shoulder height so that participants could not see their hand or the palm board when making their settings.

\section{Procedure}

We positioned people's hand randomly at one of four different angles $\left(8^{\circ}, 16^{\circ}, 24^{\circ}\right.$, and $\left.32^{\circ}\right)$, and participants verbally indicated at what orientation they felt like the flat piece of wood on which their hand rested was oriented. The axis of rotation of the hand was along the horizontal plane. Participants maintained their hand orientation while we took the digital reading of orientation and prior to having their hand repositioned to the next angle. Participants were randomly assigned to eyes open and eyes closed conditions. We used eyes open and eyes closed conditions similar to Shaffer, Taylor, McManama, et al. (2016) in order to measure any possible contribution that vision may add, as vision is typically used to stabilize the hand.

\section{Results}

We first compared estimations of the orientations of the hand between eyes open and eyes closed conditions. We performed four independent-samples $t$ tests for each of the angles comparing the eyes open and eyes closed conditions, using a Bonferroni correction of $p=.05 / 4=.0125$ for each analysis for performing the multiple $t$ tests. We found no statistical differences across conditions for any of the angles between eyes open and eyes closed conditions, $8^{\circ}: t(18)=-0.36, p=$ $724 ; 16^{\circ}: t(18)=-1.74, p=.1 ; 24^{\circ}: t(18)=-0.86, p=.404$; $32^{\circ}: t(18)=-1.89, p=.075$. Therefore, for the remaining analyses we combined eyes open and eyes closed conditions. Table 1 shows means and standard deviations for each of the four angles across eyes open and eyes closed conditions.

\section{Verbal estimates of free hand orientation are greater than actual orientations}

We then compared people's estimates of where their hand was oriented to the actual orientation at each of the four angles with four one-sample $t$ tests using a Bonferroni correction of $p=.05 / 4=.0125$ for each analysis to correct for performing multiple $t$ tests. We found that people significantly overestimated the orientation of their hand across all angles, except for the shallowest $-8^{\circ}$ angle: $8^{\circ}: t(19)=2.22, p=.039$; $16^{\circ}: t(19)=2.85, p=.01$, Cohen's $d=0.64 ; 24^{\circ}: t(19)=4.04$, $p=.001$, Cohen's $d=0.9 ; 32^{\circ}: t(19)=6.99, p<.001$, Cohen's $d=1.56$.

\section{The gain of the overestimates of free hand orientation} is similar to that of visually perceived surface orientation

To test the gain (across eyes open and eyes closed conditions) of people's estimates of the angles at which they thought their hand was oriented, we plotted the estimates for each of the 4 different angles for each participant. We then calculated and recorded the slope for each participant, and performed a onesample $t$ test comparing the gain to 1.5 , the gain that has been found for verbal estimates of palm board orientation and visually perceived near and far surfaces and for both geographical slopes outdoors as well as man-made slopes studied in laboratories. We found that the gains of our participants $(M$ $=1.67, S D=0.59)$ were statistically no different than a gain of $1.5, t(19)=1.28, p=.215$.

Table 1 Means and standard deviations for verbal estimates of all four inclinations across eyes open and eyes closed conditions

\begin{tabular}{lllll}
\hline Angle & $8^{\circ}$ & $16^{\circ}$ & $24^{\circ}$ & $32^{\circ}$ \\
\hline Mean & $13.75^{\circ}$ & $26.7^{\circ}$ & $39.25^{\circ}$ & $54.1^{\circ}$ \\
Standard deviation & $\left(11.57^{\circ}\right)$ & $\left(16.78^{\circ}\right)$ & $\left(16.88^{\circ}\right)$ & $\left(14.14^{\circ}\right)$ \\
\hline
\end{tabular}




\section{Discussion}

Our work shows that when people gave verbal estimates of hand orientation, they overestimated hand pitch orientation by a factor of $\sim 1.67$, and this is similar to that seen for verbal estimates of visually perceived slanted surfaces (Bhalla \& Proffitt, 1999; Creem-Regehr, Gooch, Sahm, \& Thompson, 2004; Durgin, Hajnal, et al., 2010; Durgin, Li, et al., 2010; Durgin \& Li, 2011; Hajnal et al., 2011; Li \& Durgin, 2010; Proffitt et al., 1995; Shaffer \& Flint, 2011; Shaffer et al., 2014; Stigliani, Li, \& Durgin, 2013). This suggests that the estimates of a freely moving hand and verbal estimates of ramps and hills reflect the same underlying perceptual representational system. The similarity in gains across tasks is indicative of a general overestimation of slanted surfaces.

This extends and is consistent with the work of $\mathrm{Li}$ and Durgin (2012, Experiment 1-orientation production task) who found that their participants overestimated their hand pitch orientation by approximately the same amount as previous studies have shown people verbally overestimate visually slanted surfaces, as shown by reverse plotting their data. Li and Durgin's participants set their hand too low to match a verbally given angle, consistent with participants in the current experiment who verbally overestimated their hand pitch orientation. This is also consistent with the proprioception calibration hypothesis that proposes that similar biases exist in both verbal estimates of visually perceived slant and proprioceptively perceived hand orientation (Li \& Durgin, 2012). These authors concluded that perceptual coding for action purposes doesn't have to be accurate, as long as both vision and proprioception are misperceived in the same way. Our results are consistent with this.

\section{Experiment 2}

Experiment 2 had three purposes. First, we wanted to measure free hand estimates while participants were looking at the slanted surface to which they were orienting their hand. Second, we wanted to directly test proprioceptively perceived hand orientation and verbal estimates of visually perceived surface orientation in the same experiment with the same participants. So after first orienting their freely oriented hand to the surface of the ramp, half of the participants verbally estimated the visually perceived orientation of the ramp, and the other half of participants verbally estimated the orientation of their unseen hand. Third, and most importantly, this allowed us to test whether verbal estimates of the orientation of a ramp are a product of, or may be predicted from, free hand orientation and verbal estimates of free hand orientation by directly comparing verbal estimates of the hand to verbal estimates of the ramp while controlling for the task that is being been used.

\section{Method}

\section{Participants}

Forty undergraduates (14 male) from The Ohio State University at Mansfield participated in fulfillment of an Introductory Psychology requirement. None had participated in Experiment 1.

\section{Materials}

We created a wooden ramp by attaching two pieces of wood (1 m $\times 1 \mathrm{~m}$ ) with a hinge. One piece of wood always stayed on the ground while the other was the portion of the ramp that was adjusted. Four precut rods were used to hold the slanted portion of the ramp at the four different angles of inclination $\left(8^{\circ}, 16^{\circ}, 24^{\circ}\right.$, and $32^{\circ}$ ). The same vertical screen blocking participants' view of their hand used in Experiment 1 was used in Experiment 2.

\section{Procedure}

Each participant stood 1 meter from the base of the ramp. Participants were told to first adjust the orientation of their hand to make it parallel with the slope of the ramp. Participants were encouraged to freely bend their hand and forearm at the elbow and wrist in any way they chose in order to do this. Participants were told when their hand was horizontal it was at $0^{\circ}$ and when their hand was vertical or straight up and down it was at $90^{\circ}$. Participants were then asked, depending on the condition to which they were assigned, to either verbally estimate the orientation of the ramp or estimate the orientation of their hand. Participants closed their eyes while the researcher moved the ramp from one angle to the next, including when it was first moved from horizontal (or flat on the ground) to the first angle, and kept their hand/forearm in the previous position to minimize the effect of anchoring (see Shaffer et al., 2014; Shaffer et al., 2015) similar to Coleman and Durgin (2014). Both groups made their estimates in degrees from horizontal. A digital reading was taken of the indicated orientation of their hand, and participants' verbal estimates were also recorded.

\section{Results}

\section{Free hand orientation}

One participant was removed from the estimate the orientation for the ramp condition as when the ramp was set to $8^{\circ}$, as they clearly did not understand the task. they estimated it to be at $0^{\circ}$, and when the ramp was set to $16^{\circ}$, they estimated it to be $15^{\circ}$ steeper than when the ramp was set to $24^{\circ}$. This participant indicated after their estimations that they were confused about the task. Means and standard deviations of free hand orientation are shown in Table 2. 
Table 2 Means (standard deviations) for free hand orientations of all four inclinations for both estimating the orientation of the hand and the ramp conditions

\begin{tabular}{lllll}
\hline Angle & $8^{\circ}$ & $16^{\circ}$ & $24^{\circ}$ & $32^{\circ}$ \\
\hline Hand & $12.93^{\circ}\left(9.03^{\circ}\right)$ & $23.48^{\circ}\left(12.05^{\circ}\right)$ & $32.66^{\circ}\left(11.4^{\circ}\right)$ & $41.6^{\circ}\left(13.09^{\circ}\right)$ \\
Ramp & $13.85^{\circ}\left(6.74^{\circ}\right)$ & $25.33^{\circ}\left(8.01^{\circ}\right)$ & $33.64^{\circ}\left(7.04^{\circ}\right)$ & $40.76^{\circ}\left(6.54^{\circ}\right)$ \\
\hline
\end{tabular}

\section{Gains of free hand orientation in both conditions are close to 1}

To test the gain of where participants oriented their hand to the ramp across all four angles, we plotted the free hand orientations to each of the four different angles for each participant and for each condition. We then calculated and recorded the slope for each participant in each condition. Best fit lines accounted for free hand orientation for the hand and ramp conditions very well, $R^{2}=.988$ and .999 , respectively. We then performed two one-sample $t$ tests to test the gains relative to 1 , the gain one would expect if free hand perception were calibrated well to the actual angle of slanted surfaces, as has been found previously, especially to near surfaces. We used a Bonferroni correction of $p=.05 / 2=.025$ for each analysis to correct for performing multiple $t$ tests. We found no statistical difference in gains between the conditions where participants estimated either their hand or the ramp and a gain of 1: Hand: $t(18)=2.33, p=.031, M=1.18, S D=0.33$; Ramp: $t(19)=$ $1.49, p=.152, M=1.11, S D=0.34$.

\section{Verbal estimates of the hand when orienting it to the ramp}

Means and standard deviations for verbal estimates of both hand and ramp orientation are shown in Table 3. To test the gain of verbal estimations of where participants oriented their hand to the ramp relative to the orientation at which their hand was actually oriented across all four angles, we plotted verbal estimates of the free hand orientations against actual free hand orientation to each of the four different angles for each participant. We then calculated and recorded the slope for each participant, and performed a one-sample $t$ test comparing the gain to 1.67, the gain found for verbal estimates of the hand in Experiment 1, and found no statistical difference in gain between verbal estimates of the orientation at which participants estimated their hand relative to their actual hand orientation

Table 3 Means (standard deviations) for verbal estimates of hand and ramp orientations for all four inclinations

\begin{tabular}{lllll}
\hline Angle & $8^{\circ}$ & $16^{\circ}$ & $24^{\circ}$ & $32^{\circ}$ \\
\hline Hand & $17.5^{\circ}\left(8.66^{\circ}\right)$ & $33.25^{\circ}\left(8.47^{\circ}\right)$ & $47.25^{\circ}\left(11.3^{\circ}\right)$ & $63.75^{\circ}\left(12.66^{\circ}\right)$ \\
Ramp & $18.37^{\circ}\left(8.04^{\circ}\right)$ & $36.47^{\circ}\left(10.09^{\circ}\right)$ & $52.89^{\circ}\left(14.37^{\circ}\right)$ & $63.84^{\circ} \backslash\left(13.06^{\circ}\right)$ \\
\hline
\end{tabular}

when looking at the ramp and a gain of $1.67, t(19)=-0.86, p=$ $.398, M=1.56, S D=0.55$.

\section{Verbal estimates of the ramp when orienting the free hand to it}

To test the gain of verbal estimations of the orientation the ramp was placed relative to the actual orientation of the ramp across all four angles, we plotted verbal estimates of the ramp orientations against actual ramp orientation to each of the four different angles for each participant. We then calculated and recorded the slope for each participant. The mean gain was $1.91(S D=0.55)$.

Actual free hand orientation and verbal estimates of free hand orientation provide an excellent fit to the verbal estimates of the ramp

In Experiment 1, the gain of verbal estimates of the hand was 1.67. In Experiment 2, the gain of the hand orientation when asked to estimate the hand was 1.18. If free hand estimates were calibrated well to verbal estimates of the ramp, then we should expect that the verbal gain we see in Experiment 1 for the hand itself multiplied by the gain of the hand to the ramp (or the difference between the hand and the ramp) when they are matched should be equal to the verbal estimates of the ramp when orienting the hand to it. That is, 1.67 times the orientation of the hand when oriented to the ramp (1.18), should be equal to $\sim 1.91$ times the orientation of the ramp. The verbal estimates of the free hand orientation found in Experiment 1 multiplied by the orientation of the hand when matched to the ramp is equal to 1.97 , almost exactly equal to 1.91, the gain of the verbal estimates of the ramp found in Experiment 2. That is, the verbal estimates of free hand orientation and the hand orientation gain when matching the hand to the ramp predicts a verbal gain close to two for Experiment 2 , which is what we found and is also shown in Table 3.

\section{Discussion}

In Experiment 2, we first examined what would happen to proprioceptively perceived free hand orientation estimates when participants were matching their hands to a distal slanted surface. We found that free hand orientation was well calibrated to a surface $1 \mathrm{~m}$ away from the observers. We also found that verbal estimates of hand orientation when orienting the free hand to a distally located ramp were statistically no different from verbal estimates of a free hand when looking at the ramp and adjusting the free hand to different orientations of the ramp. We also found that verbal estimates of different surface orientations of the ramp were predicted well by a model based on the product of actual free hand orientation when looking at a slanted surface and verbally estimated free hand orientation. 


\section{Conclusions}

We have shown that verbal estimation of free hand orientation is consistent with verbal estimates of visually perceived slanted surfaces. We have also shown that verbal estimates of visually perceived slanted surfaces out of reach may be parametrically estimated by a combination of perceived hand orientation and actual hand orientation to a slanted surface. Our work is consistent with the proprioception calibration hypothesis that proposes that similar biases exist in both verbal estimates of visually perceived slant and proprioceptively perceived hand orientation $(\mathrm{Li}$ $\&$ Durgin, 2012). Here, both vision and proprioception were misperceived in the same way. Thus, free hand and verbal estimates may be overestimates of actual surface orientation. However, as long as we overestimate our hand and the ramp/ hill in the same way (by the same magnitude), our actions will be aligned the way they need to be in order to appropriately interact with surfaces. Although it has been previously shown that free hand orientation is well calibrated to surfaces within reach, we have shown that even for slanted surfaces out of reach, free hand orientation and perception provide a reliable means for estimating verbal estimates of visually perceived slanted surfaces. Li and Durgin (2011) plotted free hand measures to outdoor hills by verbal estimates and showed that estimates of far surfaces like outdoor hills might be understood as representing the physical orientation of a near surface that would be judged equivalently — something they called a near surface equivalent. Our work extends this by showing that one might be able to predict verbal estimates of far surfaces (e.g., outdoor hills) by taking free hand orientation and the observers' perception of the orientation of their hand into consideration. This may also help to explain the results of studies investigating haptic and visual perception of outdoor hills (e.g., Bridgeman \& Hoover, 2008; Shaffer et al., 2014) that have found forearm or free hand estimates that overestimate the actual surface orientation of the hill (by a factor of $\sim 2$ ), but not as much as verbal estimates do.

So how might the results of what we found here translate to pedal perception of ground surface slant? Hajnal et al. (2011) had participants stand on a ramp that was placed at different inclines and give verbal estimates of the incline while they did this. That group then gave verbal estimates of the slope while they were not standing on it, but at the base looking at it. A different group stood on the inclines and gave free hand estimates. The proprioceptive estimates pretty well matched the verbal estimates while standing on the incline. We feel that if the participants in that study matched their foot to the incline and were then asked at what orientation their foot was placed, and another group of participants was asked to match their foot to the incline and also asked to verbally estimate the ramp, we would find the same relationship between the perception of "free foot" orientation and the verbal estimation of ramps that we found in the present work. Evidence in support of this comes from work where observers made estimates of "stand-on-able-ness" of a surface (Hajnal, Wagman, Doyon, \& Clark, 2016). They found that estimates of whether one could stand on a surface were equivalent whether the estimation was made by vision or through pedal contact with that surface. The authors proposed that their findings suggest that the visual and haptic systems can detect equivalent information about the slant properties of a surface (Hajnal et al., 2016). The idea that the proprioception calibration hypothesis may be modality independent comes not only from this and our work but also from work showing that people can perceive whether an inclined surface can be stood on by viewing the surface with an object attached to the hand (Fitzpatrick et al., 1994; Malek \& Wagman, 2008; Wagman \& Hajnal, 2014a), head (Wagman \& Hajnal, 2016), or foot (Wagman \& Hajnal 2014b). These results suggest that the perception of inclined surfaces is modality independent, and that the hand, head, or foot all give us valuable and consistent information about pedal interactions with ground surface slant. Therefore, the exaggeration of slant shown in our and others' work, and the way hand proprioception is calibrated to visual perception of surface slant, points to a single modality-independent representation of the perception of slanted surfaces generally that may serve to guide us with interactions with those surfaces.

Author Note We would like to thank Eric McManama, Allyson Thomas, and Echoe Smith for their assistance in helping to collect data.

\section{References}

Bhalla, M., \& Proffitt, D. R. (1999). Visual-motor recalibration in geographical slant perception. Journal of Experimental Psychology: Human Perception and Performance, 25, 1076-1096.

Bridgeman, B., \& Hoover, M. (2008). Processing spatial layout by perception and sensorimotor interaction. Quarterly Journal of Experimental Psychology, 61, 851-859.

Coleman, A., \& Durgin, F. H. (2014). Egocentric reference frame bias in the palmar haptic perception of surface orientation. Psychonomic Bulletin \& Review, 21, 955-960.

Creem, S. H., \& Proffitt, D. R. (1998). Two memories for geographical slant: Separation and interdependence of action and awareness. Psychonomic Bulletin \& Review, 5, 22-36. doi:10.3758/BF03209455

Creem-Regehr, S. H., Gooch, A. A., Sahm, C. S., \& Thompson, W. B. (2004). Perceiving virtual geographical slant: Action influences perception. Journal of Experimental Psychology: Human Perception and Performance, 30, 811-821.

Durgin, F. H., Hajnal, A., Li, Z., Tonge, N., \& Stigliani, A. (2010). Palm boards are not action measures: An alternative to the two-systems theory of geographical slant perception. Acta Psychologica, 134, 182-197.

Durgin, F. H., \& Li, Z. (2011). Perceptual scale expansion: An efficient angular coding strategy for locomotor space. Attention, Perception \& Psychophysics, 73, 1856-1870.

Durgin, F. H., \& Li, Z. (2012). Spatial biases and the haptic experience of surface orientation. In A. El Saddik (Ed.), Haptics rendering and applications (pp. 75-94). Rijeka, Croatia: InTech. 
Durgin, F. H., Li, Z., \& Hajnal, A. (2010). Slant perception in near space is categorically biased: Evidence for a vertical tendency. Attention, Perception \& Psychophysics, 72, 1875-1889.

Fitzpatrick, P., Carello, C., Schmidt, R. C., \& Corey, D. (1994). Haptic and visual perception of an affordance from upright posture. Ecological Psychology, 6, 265-287.

Hajnal, A., Abdul-Malak, D. T., \& Durgin, F. H. (2011). The perceptual experience of slope by foot and by finger. Journal of Experimental Psychology: Human Perception and Performance, 37, 709-719.

Hajnal, A., Wagman, J. B., Doyon, J. K., \& Clark, J. D. (2016). Perception of Stand-on-ability: Do geographical slants feel steeper than they look? Perception, 45, 768-786.

Hecht, H., Shaffer, D. M., Keshvarz, B., \& Flint, M. (2014). Slope estimation and viewing distance of the observer. Attention, Perception, \& Psychophysics, 76, 1729-1738.

Li, Z., \& Durgin, F. H. (2010). Perceived slant of binocularly viewed large-scale surfaces: A common model from explicit and implicit measures. Journal of Vision, 10, 1-16.

Li, Z., \& Durgin, F. H. (2011). Design, data, and theory regarding a digital hand inclinometer: A portable device for studying slant perception. Behavior Research Methods, 43, 363-371. doi:10.3758/s13428010-0047-7

Li, Z., \& Durgin, F. H. (2012). Manual matching of perceived surface orientation is affected by arm posture: Evidence of calibration between proprioception and visual experience in near space. Experimental Brain Research, 216, 299-309.

Malek, E. A., \& Wagman, J. B. (2008). Kinetic potential influences visual and remote haptic perception of affordances for standing on an inclined surface. Quarterly Journal of Experimental Psychology, 61, 1813-1826.

Proffitt, D. R., Bhalla, M., Gossweiler, R., \& Midgett, J. (1995). Perceiving geographical slant. Psychonomic Bulletin and Review, 2, 409-428.

Shaffer, D. M., \& Flint, M. (2011). Escalating slant: Increasing physiological potential does not reduce slant overestimates. Psychological Science, 22, 209-211.
Shaffer, D. M., \& McManama, E. (2015). Remote haptic perception of slanted surfaces shows the same scale expansion as visual perception. Attention, Perception, \& Psychophysics, 77, 948-952.

Shaffer, D. M., McManama, E., Swank, C., Williams, M., \& Durgin, F. H. (2014). Anchoring in action: Manual estimates of slant are powerfully biased toward initial hand orientation and are correlated with verbal report. Journal of Experimental Psychology: Human Perception and Performance. doi:10.1037/a0036217

Shaffer, D. M., McManama, E., \& Durgin, F. H. (2015). Manual anchoring biases in slant estimation affect matches even for near surfaces. Psychonomic Bulletin \& Review, 22(6), 1665-1670.

Shaffer, D. M., Taylor, A., McManama, E., Thomas, A., Smith, E., \& Graves, P. (2016). Palm board and verbal estimates of slant reflect the same perceptual representation. Attention, Perception, \& Psychophysics, 78, 663-673. doi:10.3758/s13414-015-1029-8

Shaffer, D. M., Taylor, A., Thomas, A., Graves, P., Smith, E., \& McManama, E. (2016). Pitching people with an inversion table: Estimates of body orientation are tipped as much as those of visual surfaces. Attention, Perception, \& Psychophysics, 78, 700-706. doi:10.3758/s13414-015-1019-x

Stigliani, A., Li, Z., \& Durgin, F. H. (2013). Humans have precise knowledge of familiar geographical slants. Journal of Experimental Psychology: Learning, Memory, and Cognition, 39, 1966-1973.

Taylor-Covill, G. A. H., \& Eves, F. F. (2013). The accuracy of "haptically" measured geographical slant perception. Acta Psychologica, 144, 444-450.

Wagman, J. B., \& Hajnal, A. (2014a). Task specificity and anatomical independence in perception of properties by means of a wielded object. Journal of Experimental Psychology: Human Perception and Performance, 40, 2372-2391.

Wagman, J. B., \& Hajnal, A. (2014b). Getting off on the right (or left) foot: Perceiving by means of a rod attached to the preferred or nonpreferred foot. Experimental Brain Research, 232, 3591-3599.

Wagman, J. B., \& Hajnal, A. (2016). Use your head! Perception of action possibilities by means of an object attached to the head. Experimental Brain Research, 234, 829-836. doi:10.1007/s00221$015-4509-0$ 